\title{
Continuously Monitoring Foetal ECG using Mobile Telemedicine Sensor Network
}

\author{
Mahmoud Ahmed Suliman Ali \\ Department of electrical \& electronic Engineering , faculty of Engineering science, Nyala University, Nyala, Republic of the Sudan \\ Email: mahmoud_as@hotmail.com
}

Received 2013

\begin{abstract}
Foetus ECG monitoring based on Bluetooth portable devices promise to provide an efficient, accurate, and economic way to monitor foetus health outside the hospital. In this paper we discuss a new idea in biomedical field may be useful for Medical services. The idea is deliver the status of patent to any location within the coverage of cellular networks, such as the global system for mobile (GSM) communications. Pregnancy women from a rural area just like rural in Sudan (where they haven't transportation to a hospital), could be given a routine check by mobile phone without having to commute regularly to a hospital. Routine inspections and monitoring could be done while the pregnancy women is at home, traveling(nomadic), at work, or at leisure, thereby relieving resources for more demanding hospital cases. Advances in mobile technology have made wireless telemedicine more practical both within hospitals and globally. The Bluetooth system with low cost the poor women can use and also has low electromagnetic waves, it is healthy in use (no risk).
\end{abstract}

Keywords: FECG; Bluetooth; Mobile System and GPRS

\section{Introduction}

Foetal electrocardiogram is a very useful technique for monitoring foetus in side the mother's abdominal. Recently there is some research expected the Continuous foetal heart rate (FHR) monitoring during labor may utilized $85 \%$ of labor episodes in the United States and represents the standard of care[1], although there is scant evidence to demonstrate that the use of the technology improves newborn or maternal outcomes.

Encouraging data demonstrate that intrapartum foetal electrocardiogram (fECG) analysis can reduce newborn acidemia, hypoxic ischemic encephalopathy, and cesarean deliveries. However, the only clinically available device for fECG analysis-the STAN monitor from Neoventa (Moindal, Sweden)—requires an invasive foetal scalp electrode (FSE), limiting its use to a subset of pregnant women who are laboring with ruptured membranes and a dilated cervix. The potential utility of noninvasive fECG for foetal evaluation is significant. However, to date [2], there has been no systematic study proving that fECG can be extracted non-invasively without distorting important clinical parameters, such as the ST segment. Prior reports have shown the capacity to measure FHR using electrodes on the maternal abdomen, but none have demonstrated the capacity to accurately record the fECG waveform with sufficient fidelity to evaluate the morphology.
FECG is project may be developed to be continuously mentoring in small portable devices and cheap, suitable for the poor family. Recently Sensor Mobile telemedicine is a newly emerging branch of medical service, means delivered Medical services to any location within the coverage of cellular networks. This project review on Sensor mobile telemedicine used for Sensing of Electrocardiogram (ECG) data, Electrocardiogram is information about human heart, and then the information detected by sensor is electrical signal generated by heart. Known as The standard FECG signal consists of six peak signals each defined with a different letter, the P, Q, R, S, $\mathrm{T}$ and $\mathrm{U}$ peaks [1]. The Sensor Mobile telemedicine has the potential to improve patients' quality of life by allowing them to move around freely while undergoing continuous heart monitoring and to reduce healthcare costs associated with prolonged hospitalization, treatment and monitoring.

\section{Literature Review}

Foetus ECG monitoring is a technique for obtaining important information's about the condition of the foetus during pregnancy and labour by measuring electrical signals generated by the foetal heart as measured from multi-channel potential recordings on the mother body surface or hearing foetus heart beat, because Being born is one of the most crucial events in our life. Historically 
In 1958 using an electrode placed through the maternal abdomen on the foetus. [2]. There are two situations for which FHR provides important information about the condition of the foetus. It is known that FHR monitoring is able to distinguish between the so called reactive foetus and the so called non-reactive. To achieve the goal using advance signal processing to extract FOETAL signal from maternal signal \& other noise to gain a bur FECG Signal .

The normal Electrocardiogram signal is (PQRST) signal.

The PQRST complex is an electric signal.

Produced by the contraction of the heart's muscle called myocardium. It is composed of three parts: the Pwave reflects the contraction of the auricles; the QRScomplex is associated with the contraction of the ventricles. Due to the magnitude of the R-wave, it is extremely reliable; the T-wave, which corresponds to the depolarization phase which follows each heart contraction. The delay associated to the R-R interval leads to the heartbeats frequency. Traditionally way of providing medicine services is to transmit pregnancy women to a hospital or to transmit biomedical signals from a pregnancy women to a hospital using "landlines," such as the Public Switched Telephony, the last is advance for transmit the signal to hospital called telemedicine. [3] Network (PSTN) and the Integrated Services Digital Network Most current telemedicine applications are limited to communications between fixed locations, often with conventional handsets. The adoption of mobile technology has led to new $\mathrm{m}$-Health applications in health-care provision [4]. Although face-to-face consultations between a clinician and pregnancy women will never be replaced, there are medical cases that can be managed more efficiently by adopting wireless telemedicine. Potential mobile applications include remote routine checkups, emergency and rescue situations, and sports science physiological measurements [10]. Medical services can now be delivered to any location within the coverage of cellular networks, such as the global system for mobile (GSM) communications[9]. Pregnancy women from a rural area just like rural in Sudan could be given a routine check by mobile phone without having to commute regularly to a hospital. Routine inspections and monitoring could be done while the pregnancy women is at home, traveling, at work, or at leisure, thereby relieving resources for more demanding hospital cases. Advances in mobile technology have made wireless telemedicine more practical both within hospitals and globally [3].

\section{Design Considerations}

The desired end product is a sensor mobile telemedicine processor to collect sample of combine ECG signal (FECG+MECG+Noise ) and transmit up to four channels of biomedical signals via a cellular network.
The design of the processor must be "future-proofed," so that future processing and memory upgrades can be achieved simply by software changes. The hardware is, therefore, implemented with a programmable logic device (PLD), and a programmable, multichannel analogto-digital converter (ADC) allows data to be handled for high-bandwidth third- generation (3G) networks.

The connection between the processor and the mobile telephone is implemented with a Bluetooth master-slave link.

Alignment between the processor and the telephone is, therefore, not critical, unlike with an infrared link.

The connection between the telephone and the hospital uses the General Packet Radio Service (GPRS) [8], which allows simultaneous data and voice transmission.

The Figure 1 has shown the following links,

1) Short-range Bluetooth link between pregnant women and doctor telephone.

2) GPRS to a base station and to other mobile telephones;

3) land lines (PSTN) and an Internet service provider (ISP) to a hospital server and database.

4) LAN to clinicians (doctor laptop).

\section{Limitation of the System}

Bluetooth 128 bit authentication key and 8 bit encryption key [5]. WLAN wired equivalent privacy (WEP) protocol with RC4 encryptions algorithm [8] .GPRS three-tier security with A3 algorithm for user authentication A8 ciphering key generating algorithm and A5 ciphering Algorithm for data Encryption.

3G f8 UMTS confidentiality algorithm and f8 UMTS Integrity Algorithm

The rationale for choosing the components of this system is now explained. The methodology includes consideration of a combination of wireless techniques, particularly the exploitation of cellular networks, types of clinical data for transmission, and system memory storage.

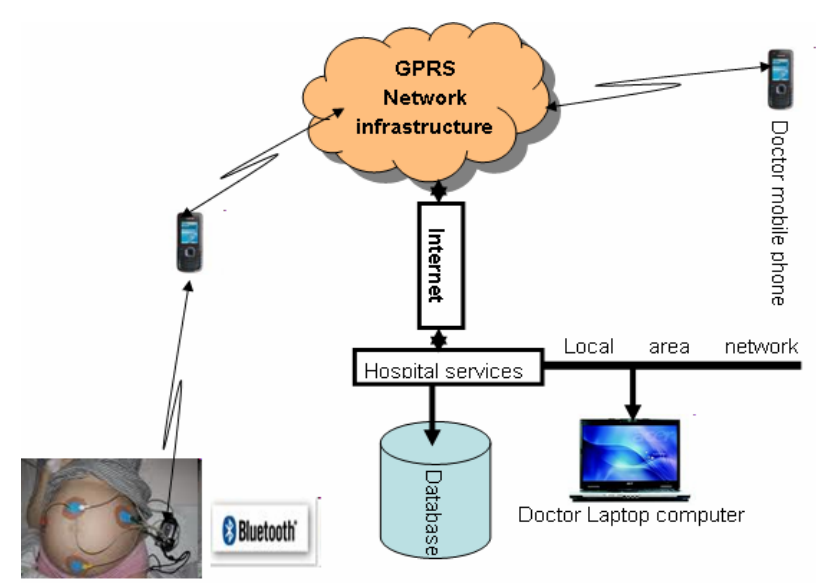

Figure 1. Diagram of the Bluetooth medical service. 
For Bluetooth Data Transmission we choose BlueSmirf module provided by Sparkfun Electronics [6]. It is a class 1 model that has an approximate range of 100 meters. The asynchronous data from the electrode are delivered to the BlueSmirf Bluetooth module on the serial port. The Bluetooth module is configured as a slave and the mobile phone is considered to be functioning as a master. The signal acquisition unit sends data to the Bluetooth module, which transmits data continuously, in blocks of FECG samples plus, the data are sent as raw binary bytes.

The electrocardiogram data for human assumed to be Familiar for all researchers of biomedical signal, the Table 1 Below described all types of biomedical data.

In the case of the design of sensing devices, focus on Bluetooth Telemedicine Processor for Multichannel Biomedical Signal Transmission via Mobile Cellular Networks which samples signals from sensors on the patient [11], It then transmits digital data over a Bluetooth link to mobile telephone and then through system to the doctor .The system that uses General Packet Radio Service (GPRS). The used Bluetooth is a universal shortrange low-power radio protocol operating in the unlicensed industrial, scientific, and medical frequency band. It allows both data and voice connections, the modulation technique is Gaussian frequency-shift keying, with transmission at a rate of $1 \mathrm{Msymbol} / \mathrm{s}$ on one of 79 channels with $1-\mathrm{MHz}$ spacing in the $2.402-2.480-\mathrm{GHz}$ band. Bluetooth uses a spread-spectrum frequency hopping [7] connection with a rate of 1600 hops/s and its radio transceivers are categorized in three power classes, as shown in Table 2 below.

Table 1. Biomedical data.

\begin{tabular}{lcc}
\hline \multicolumn{1}{c}{ Biomedical data } & Type & Typical file size \\
\hline ECG Record & Electrical signal & $100 \mathrm{~KB}$ \\
Electronic stethoscope & Audio & $100 \mathrm{~KB}$ \\
X-ray & Still image & $1 \mathrm{MB}$ \\
30s of ultrasound image & Moving image & $10 \mathrm{MB}$ \\
\hline
\end{tabular}

Table 2. Power class and range.

\begin{tabular}{ccc}
$\begin{array}{c}\text { Power } \\
\text { Class }\end{array}$ & $\begin{array}{c}\text { Maximum Outputs } \\
\text { Power }\end{array}$ & $\begin{array}{c}\text { Typical } \\
\text { Range }\end{array}$ \\
\hline 1 & $100 \mathrm{~mW}(20 \mathrm{dBm})$ & $100 \mathrm{~m}$ \\
2 & $25 \mathrm{~mW}(4 \mathrm{dBm})$ & $20 \mathrm{~m}$ \\
3 & $1 \mathrm{~mW}(0 \mathrm{dBm})$ & $10 \mathrm{~m}$ \\
\hline
\end{tabular}

The hardware is, therefore, implemented with a programmable logic device (PLD), and a programmable, multichannel analog-to-digital converter (ADC) allows data to be handled for high-bandwidth third-generation (3G) net- works. The connection between the processor and the mobile telephone is implemented with a Bluetooth master-slave link, the figure below show all mobile telemedicine system and the alignment between the Bluetooth processor and mobile telephone.

\section{Materials and Method}

FECG signal is not ready signal to be taken ,it needs some extraction method to prepare before sending, In this study there are two models, presented the model for signal taken by Thoracic Electrodes $x_{1}(t)$ which record Thoracic Electrocardiogram (TECG), represented by $s_{1}(t)$ and the model for signal taken by Abdominal Electrodes $x_{2}(t)$ which record Abdominal Electrocar-diogram (AECG) represented by $s_{2}(t)$

As mentioned above the foetal ECG is very weak among the maternal ECG and noise. For this reason the Significant recent advances in the field of statistical signal processing should be brought to the attention of the biomedical engineering community. Algorithms have been proposed to separate multiple signal sources based solely on their statistical independence, instead of the usual spectral differences. These algorithms promise to:

- lead to more accurate source modeling,

- More effective artifact rejection algorithms

Independent Component Analyses (ICA) \& Blind Source Separation (BSS)(see Figure 2 Below ) are becoming very popular for extraction FECG signal from the AECG. The use of these techniques for extraction of FECG in noninvasive methods just need amount of electrode for record signal from abdominal.

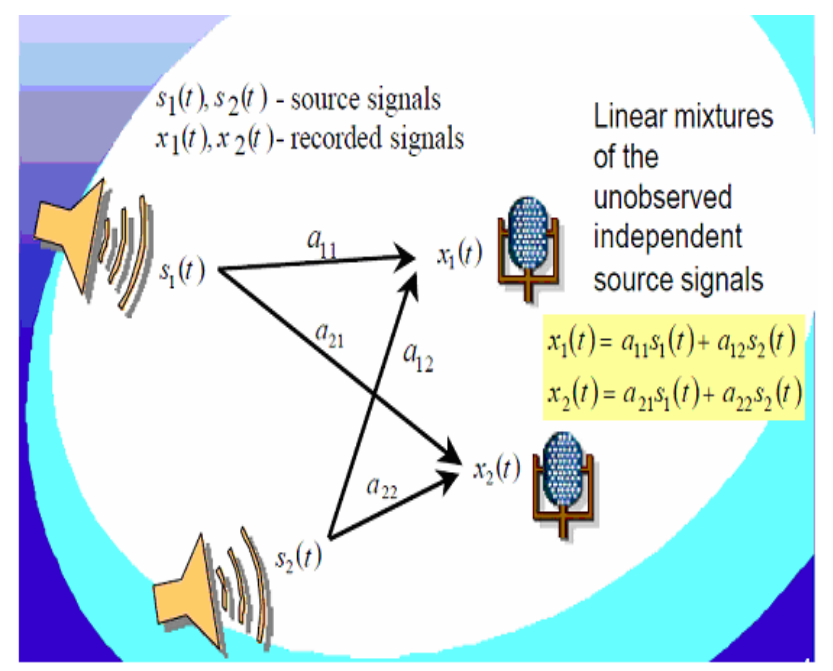

Figure 2. Independent component analysis ( ICA). 


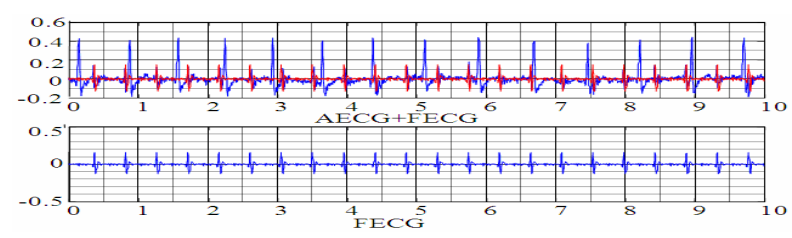

Figure 3. Shows the abdominal records and pure foetal signals.

Already the records of the maternal Thoracic Electrocardiogram (TECG) signals are known and the records of the Abdominal Electrocardiogram (AECG) which contains foetal ECG + maternal ECG represent the complex part.

\section{Results}

The maternal ECG signal interference was canceled from the foetal heart ECG signal .ECG signals are given as an input and is simulated using MATLAB. The results of programs are shown in the Figure 3 . These results represent the raw data of PQRST and U complex signal recorded from maternal abdominal and pure PQRST complex signal for Fetus after extraction, this results should be appeared at doctor laptop computer or mobile phone

\section{Discussion}

Figure 3 in results above contains two graphs. The upper graph shows AECG and FECG recombined after being extracted. In comparing these two signals we can see the region of FECG in AECG signal. The lower graph shows pure FECG. The amplitude of QRS of FECG is about 30 microvolt while that of MECG is about 150 microvolt. These values may agree to the value of QRS amplitude. The obtained result shows the effectiveness of the proposed algorithm.

\section{Conclusions}

It can be assumed from this study that the goal of Sensor mobile telemedicine is to record real accurate electrocardiogram data and encapsulate then transmit to the target safety without any interference. It is important to understand the actual though process over several wireless systems of individual technique which have different attachment styles. It is easy to transfer this idea to sense and monitoring one of very important issue that is foetal electrocardiogram (FECG), but the weakness of FECG signal need more security technique to avoid interference of the noises.

The algorithm used in this study is very simple and not complex. The performance and validity of the proposed algorithm have been confirmed by computer simulations and experiment on real-world ECG data. The data used here is public databases widely used by the signal processing community known as SISTA/DAISY dataset. The result which was obtained appears to agree with the standard Foetal ECG signals. The Researcher recommends following this method to gain more useful results.

\section{Acknowledgements}

This work was supported in part by university of Nyala Republic of the Sudan. Many thanks to the University of Nyala for offering me the fund for this conference.

\section{REFERENCES}

[1] R. Sameni, "Extraction of Foetal Cardiac Signals from an Array of Maternal Abdominal Recordings," Sharif University of Technology, Tehran, Iran, Doctor of Philosophy thesis , July 2008

[2] A. M. J. Lenssen. "From Cardiac Electrical Activity to the ECG: A finite Element Model,” Master thesis, Eindhoven University of Technology, May 2008.

[3] N. M. Fisk, S. Bower and W. Sepulveda, "Foetal Telemedicine: Interactive Transfer of Real Time Ultrasound and Video Via ISDN for Remote Consultation,” Journal of Telemedicine and Telecare, Vol. 1, No. 1, 1995, pp. 38-44.

[4] M. F. A. Rasid and B. Woodward, "Bluetooth Telemedicine Processor for Multichannel Biomedical Signal Transmission Via Mobile Cellular Networks,” IEEE, Vol. 9, No. 1, March 2005.

[5] Noureddine BELGACEM, Fethi BEREKSI-REGUIG, “A Design of DSPIC Based ECG Signal Monitoring and Processing System,” BP 230 Tlemcen, 2003

[6] O. Krejcar, D. Janckulik, L. Motalova and K. Musil, "Real Time Processing of ECG Signal on Mobile Embedded Monitoring Stations," Second International Conference on Computer Engineering and Applications, 2010.

[7] S. Da` gtas, G. Pekhteryev, Z. Sahino ` glu, H. C am and N. Challa, "Real-Time and Secure Wireless Health Monitoring," Hindawi Publishing Corporation International Journal of Telemedicine and Applications, Article ID, 2008, 135808.

[8] J. P. Tello, O. Manjarres, M. Quijano, A. Blanco, F. Varona and M. Manrique,' Remote Monitoring System of ECG and Body Temperature Signals," IEEE LATIN AMERICA Transactions, Vol. 11, No. 1, Feb 2013.

[9] Zuckerwar, R. Pretlow, J. Stoughton and D. Baker, "Development of a Piezopolymer Pressure Sensor for a Portable Foetal Heart Rate Monitor," IEEE Trans. Biomed. Eng., Vol. 40, No. 9, 1993, pp. 963-969. doi:10.1109/10.245618

[10] M. Scanziani and M. Häusser, "Electrophysiology in the Age of Light,” Nature, 2009, Vol. 461, 15 October.doi:10.1038/nature08540

[11] E. M. Symonds, "Foetal Electrocardiographic Monitoring: Past, Present and Future--a Historical Perspective,” J Perinat Med., Vol. 14, No. 6, 1986, pp. 385-90. 